\title{
Urs Peter Schneiders Walser-Kosmos
}

\author{
Ein Laboratorium
}

Der in Biel lebende Komponist und Pianist Urs Peter Schneider hat sich mit Robert Walser über einen Zeitraum von mehr als fünfzig Jahren auseinandergesetzt. Insgesamt siebzehn seiner Werke in diversen Gattungen beziehen sich auf Walser. Dominierend sind experimentelle kompositorische Verfahren, die nur in einzelnen Fällen noch als >Vertonung im traditionellen Sinn bezeichnet werden können. Dieses Kapitel gibt Einblicke ins Kompositionslaboratorium von Urs Peter Schneider.

\section{1 $\quad$ Einleitung}

Urs Peter Schneider wurde 1939 in Bern geboren und lebt seit 1966 in Biel. Er hat Klavier am Konservatorium Bern bei Walter Lang und in Köln und Wien bei Bruno Seidlhofer studiert, dazu Komposition, ebenfalls in Bern, bei Sándor Veress. Die späten 1950er-Jahre erwiesen sich als eine äußerst fruchtbare Zeit für das Berner Konservatorium (heute Hochschule der Künste Bern): Innerhalb von knapp zehn Jahren wurden dort die Musiker Jürg Wyttenbach, Heinz Holliger, Roland Moser und eben Urs Peter Schneider ausgebildet, um vier wegweisende Komponisten zu nennen. Allen gemeinsam ist das Interesse für Literatur und ein Engagement für musikalische Qualität, das sich gegen leeres Virtuosentum, billige Effekte und Anbiederungen aller Art richtet. Alle vier wurden deshalb auch bedeutende Hochschullehrer und gaben dieses künstlerische Ethos an die nächsten Generationen weiter. Ihre größte Gemeinsamkeit allerdings bleibt, dass sie, jeder für sich, kompromisslos und eigensinnig ihren Weg gehen - und damit künstlerisch völlig unterschiedliche Richtungen einschlagen.

Nach Veress vertiefte Urs Peter Schneider sein Kompositionsstudium bei so unterschiedlichen Persönlichkeiten wie Henri Pousseur, Frederic Rzewski und Karlheinz Stockhausen. Insbesondere auf Karlheinz Stockhausen geht Schneiders Position zurück, nicht zu schreiben, was man fühlt oder innerlich hört, sondern kompositorische Strategien zu entwickeln, mit denen man zu neuen Klängen gelangt, die man sich selber gar nicht vorstellen konnte. 1968 gründete Schneider mit seiner ersten Frau, Erika Radermacher, das bis heute 
aktive Ensemble Neue Horizonte Bern, das - ähnlich den >Hausabenden < der Familie Gattiker ${ }^{1}$ und in deren Nachfolge - während Jahrzehnten die zeitgenössische Musik in Bern pflegte. Obwohl in seinen Konzertprogrammen alle wichtigen Stile vertreten sind, ist es eines der Ensembles, das schon früh systematisch die Konzeptmusik amerikanischer Prägung aufführte und in Europa bekannt machte (Schneider hat auch mit - und für - John Cage gearbeitet).

Am Konservatorium Bern begründete Urs Peter Schneider das Unterrichtsfach >Ensemble fächerübergreifend ‘, in dem er vieles von dem, was heute unter interdisziplinären Studien an diversen Kunst- und Musikhochschulen angeboten wird, mit Weitblick vorwegnahm: Bis zu seiner Emeritierung 2002 lernten die Studierenden bei ihm, in Kontexten zu arbeiten und unterschiedliche Disziplinen und Künste zu verbinden, dies sehr präzis und definiert. Das Ungefähre und Beliebige ist Urs Peter Schneider bis heute ein Graus. Auch als Pianist liebt er klare und Stellung beziehende Interpretationen; seine bevorzugten Komponisten sind Carl Philipp Emanuel Bach und vor allem Wolfgang Amadeus Mozart, »dessen höchste Komplexität bei oft harmlosester Oberfläche [ihm] Vorbild [ist], dem Stockhausen übrigens auch! «²

Ab 1973 widmete sich Schneider auch der Improvisation: Seine wilden Performances mit großen Improvisatoren wie Pierre Favre, Irène Schweizer, Maggie Nichols, Philippe Micol und John Zorn sind berühmt. Die Improvisation ist für Schneider allerdings eine völlig eigenständige Domäne, die er mit seinem Komponieren nicht in Verbindung bringt. Auch Robert Walser hat er sich nie improvisierend genähert.

\subsection{Walser-Experimente}

Bei Urs Peter Schneider, dem Suchenden nach neuen Horizonten, gibt es auch Beharrendes, feste Rituale, eine Treue zu gewissen Traditionen: Robert Walser liest er bis heute in der ersten Gesamtausgabe des Kossodo-Verlags, der u. a. wegen der Größe dieses Vorhabens Konkurs gegangen ist. Schneiders Äußeres scheint seit Jahrzehnten unverändert, mit seinen lohenden Haaren ist er schon von Weitem erkennbar. Und konsequent schreibt er seine eloquenten, witzigen und mit zahlreichen Seitenhieben versetzten Programme, Kommentare, Notizen und Briefe auf der gleichen Schreibmaschine mit engem Zeilenabstand. Kommunikationstechnologisch ist Schneider auf dem Stand von 1980 stehen

1 Vgl. Lanz: Neue Musik in alten Mauern.

2 Brief von Urs Peter Schneider an Roman Brotbeck vom 04.o9.2016. 
geblieben. So muss man Urs Peter Schneider, will man ihn etwas fragen, einen Brief schreiben. Die Distanz zum Computer und dessen Kompositions- und Notationsprogrammen ist übrigens allen eingangs erwähnten Veress-Schülern gemeinsam. Alle vier sind bis heute stolz darauf, Strukturen und Gestalten zu komponieren, die ein Computer nur schwer hätte rechnen oder bewältigen können. Für mich war das - auch als Referenz an den großen Briefeschreiber Walser - eine Einladung, mit Urs Peter Schneider in einen Briefwechsel zu treten. Sein Verhältnis zum Schriftsteller beschreibt er folgendermaßen:

Zu Walser und mir: 1) Biel-Bezug; 2) Spazieren; 3) Verwahrlosung (der ich dialektisch während meines ganzen Lebens durch »Schreib-Tugend « entgegen halte); 4) Klangfarbenreichtum, Reichtum der Artikulationen, bis hin zu Klangfarbenfindungen (die aus Strukturüberlegungen kommen, bei Walser aus Schnelligkeiten, als »Geschenke«); 5) Musik über Musik, ${ }^{3}$ bei Walser das Schreiben übers Schreiben; aber 6) ist es einfach eine Liebesbeziehung, die nicht leicht erklärt werden kann (und auch nicht muss). ${ }^{4}$

Urs Peter Schneider ist unter den Walser-Vertonern jener Komponist, der sich am experimentellsten mit dem Schriftsteller auseinandersetzt, meist mit konzeptionellen Ansätzen.

Meine zahlreichen Walser-Vertonungen sind indessen, mit Ausnahme von »Beiseit« und »Chorbuch«, nie Textvertonungen! Ich nehme seine Texte als Motto, als Strukturvorlagen (nach genauen Analysen), als zur Musik gesprochene: ich verdopple nicht das, was schon dasteht! ${ }^{5}$

Schon bei den zwei radikal gegensätzlichen und gerade deshalb auch wieder erstaunlich symmetrischen Beiseit-Vertonungen (vgl. Kap. 10.4) bricht bei Schneider dieses Konzeptionelle durch. Auch das von Schneider im Brief erwähnte Chorbuch für acht Sängerinnen und Sänger ist keine traditionelle

3 Die Wendung »Musik über Musik« geht auf Theodor W. Adorno zurück, der sie 1949 als Kritik auf Igor Strawinskys neoklassizistische Phase formuliert, dabei allerdings ex negativo Entscheidendes über dieses reflektierende Verfahren bei Walser und zahlreichen WalserVertonern, allen voran Schneider, aussagt: »Das autoritäre Prinzip des Musik über Musik Machens ist so gewandt, daß allen erdenklichen veralteten Musikformeln die Verbindlichkeit vindiziert wird, die sie historisch verloren haben und die sie erst zu besitzen scheinen, sobald sie sie nicht mehr besitzen. Zugleich wird das Usurpatorische der Autorität zynisch unterstrichen durch kleine Willkürakte, die den Hörer blinzelnd über die Illegitimität des Autoritätsanspruchs informieren, ohne doch von diesem das mindeste nachzulassen.« Adorno: Philosophie der neuen Musik, S. 188.

4 Brief von Urs Peter Schneider an Roman Brotbeck vom 08.07.2016.

5 Ebd. 
Textvertonung, obwohl der kleinfügig, aber wirksam bearbeitete Text nachvollziehbar ist, sondern eher ein Walser-Exerzitium.

Fast 6o Jahre liegen zwischen Schneiders erster Auseinandersetzung mit Walser $195^{8}$ und seiner vorläufig letzten Walser-Komposition Teich mit zwei Schwänen (2016) für Klaviertrio zu dessen 6o. Todestag. ${ }^{6}$ Als verbindendes Moment all seiner Arbeiten zu Walser sieht er die Spannung zwischen Musik und Text:

Vielleicht kann man sich zurechtlegen, dass Walser-Vertonungen (bei mir) in einer Spannung zum Text stehen, in einer Art Zerreißprobe; aber aufgefangen durch (meinen) strukturalen Humor (Non-Espressivo, Non-Hysterie, Nicht-Dramatik). ${ }^{7}$

\subsection{Die vier Bücher}

Am intensivsten ist die Präsenz von Robert Walser in Die vier Bücher, Schneiders 〉Opus Magnum<, welches das Schaffen aus 27 Jahren zusammenfasst. Die durchaus ironisch-hintersinnig gewählten Titel Liederbuch, Chorbuch, Zeremonienbuch und Orchesterbuch, die an die unzähligen Bücher im kirchlichen und profanen Bereich erinnern, suggerieren einen zufälligen Sammlungscharakter und lassen eine Art Schneider-Sammlung erwarten. Das ist eine falsche Fährte, denn jedes der vier Bücher gehorcht einem rigiden Konzept, und die Bücher als Ganzes weisen ebenfalls zahllose Querbezüge auf. Obwohl Schneider alles andere als ein serieller Komponist ist, sind die Ordnungs- und Organisationsprinzipien der Serialisten, insbesondere seines Lehrers Karlheinz Stockhausen, doch permanent präsent: Er wählt als Material musikalische >Alphabete $<$ aus, die meist eine große Spannweite aufweisen; in diesen Alphabeten nutzt er dann alle Abstufungen und alle Kombinationen unter konsequenter Vermeidung der Wiederholung; auch scheinbar ähnliches Material wird nach strengen Konstruktionsprinzipien ständig variiert. »Zudem wird im Kleinen und Großen universelle Vermittlung (Stockhausen!), möglichste Kohärenz angestrebt. « ${ }^{8}$

6 Die Walser-Werke von Urs Peter Schneider sind in verschiedenen Aufnahmen publiziert worden. Das Zeremonienbuch erschien 1984 auf einer LP in einer Interpretation mit dem Blockflötisten Conrad Steinmann. Chorbuch und Orchesterbuch erschienen 1997 auf einer Porträt-CD, die Robert Walser Trilogie I sowie die Beiseit-Vertonungen aus dem Liederbuch auf der CD Robert Walser in der Schweizer Musik. Für weitere Aufnahmen vgl. das Verzeichnis der veröffentlichten Aufnahmen von Walser-Vertonungen im Anhang.

7 Ebd.

8 Brief und Material von Urs Peter Schneider an Roman Brotbeck vom 04.09.2016. 
Dieses quasi-serielle Denken, das auf die Walser'schen Verfahren ein durchaus neues Licht wirft und ihn viel eher als Konstrukteur und Konzept-Dichter denn als frei improvisierenden Sänger und Spaziergänger versteht, betrifft alle Bereiche von Schneiders Komponieren: So besteht das Liederbuch aus 35 Liedern, die auf zehn Texten von zehn deutschen Dichtern aus zehn Jahrhunderten beruhen; Schneider entnimmt sie »aus meiner grossen Anzahl von Gedichtbänden und Anthologien vergangener Jahrhunderte«, wobei die Auswahl »nach expressiven und strukturellen Gesichtspunkten « erfolgte. ${ }^{9}$ Robert Walser, der im Liederbuch mit den beiden Beiseit-Vertonungen vertreten ist (vgl. Kap. 10.4), wird damit in einen 10oo-jährigen Kontext deutscher Literatur gestellt und von Schneider dem 19. Jahrhundert zugeordnet. ${ }^{10}$ Speziell ist Schneiders Verfahren der Mehrfachvertonung, das er zum Kompositionsprinzip erhebt.

Die Mehrfachvertonungen habe ich im Liederbuch auf die Spitze getrieben (z. B. Abruf von Arnold Leifert 13x auf denselben Text): die Musik wechselt, der Text bleibt (im Ggs. zu Schubert, zum Strophenlied)!1!

Schneider sucht also nicht nach einer einzigen, >idealen « musikalischen Lösung für einen Text, sondern schlägt verschiedenste Varianten vor, die - wie es seine Beiseit-Vertonungen zeigen - einen Text auch in ihren gegensätzlichsten Aspekten darstellen. Obwohl Schneider nicht nur bei Walser so vorgeht, trifft er doch einen wesentlichen Aspekt des die Variation so liebenden Dichters. Die Anzahl der Vertonungen pro Text ist von Schneider auch ins großformale Konzept eingebunden: Vier Texte werden nur einmal, drei werden zweimal, zwei werden sechsmal und der bereits erwähnte Abruf von Arnold Leifert wird dreizehnmal vertont. Die zehn Teile sind denn auch fast symmetrisch geordnet: $1-2-6-2-1-13-2-1-6-1$.

\subsection{Walser-Zeremonien}

Im Zeremonienbuch für ein Holzblasinstrument versammelt Schneider 25 Zeremonien auf sechs Texte deutscher Dichter aus sechs Jahrhunderten. Diese Zeremonien sind alle rein instrumental realisiert - es wird kein Text gesungen oder gesprochen -, aber die Texte bilden die strukturelle und oft

9 Schneider: Liederbuch, S. 1.

10 In der Partitur datiert Schneider Beiseit mit »ca. 1895«. Vgl. Liederbuch, S. 1. Tatsächlich ist Beiseit das erste Mal im August 1899 in der Wiener Rundschau erschienen und hat dann einige Nachdrucke, u. a. im Band Gedichte (1909/1919), erfahren.

Brief und Material von Urs Peter Schneider an Roman Brotbeck vom 04.09.2016. 
auch >semantische< Grundlage der Musik. Auch hier kommen >Mehrfachvertonungen vor, die allerdings nur als `Variationen $<$ wahrgenommen werden. Auch im Zeremonienbuch wird mit der Anzahl dieser Variationen die Großform gestaltet und eine Fast-Symmetrie erreicht $(1-5-1-12-5-1)$. Die Autoren der Texte sind:

$\begin{array}{lr}\text { Johann Faustus (ca. 1535) } & \text { 1-mal } \\ \text { Sänger von Treuchtlingen (ca. 1475) } & 5 \text {-mal } \\ \text { Wilhelm Ramler (ca. 1755) } & \text { 1-mal } \\ \text { Daniel von Reigersfeld (ca. 1655) } & \text { 12-mal } \\ \text { Robert Walser (ca. 1895) } & 5 \text {-mal } \\ \text { Ludger von Diedrichsfeld (ca. 1975) } & \text { 1-mal }\end{array}$

Da Ludger von Diedrichsfeld nirgends nachweisbar ist, dessen experimentelles Gedicht aber an eine Komposition von Schneider erinnert, vermute ich, dass Schneider selbst dieses Gedicht als ironisches Wiegenlied geschrieben hat. ${ }^{13}$ Es kann als literarische Kurzeinführung ins musikalische Denken und Schaffen von Urs Peter Schneider gelten: Aus 26 verschiedenen Wörtern wird ein Gedicht mit 12 mal 8 Wörtern, also 96 Wörtern gestaltet; grafisch wird das Gedicht in der Schreibmaschinen-Typografie dargestellt. Es zeigt anschaulich, was mit dem von Urs Peter Schneider erwähnten >strukturalen Humor< gemeint ist (vgl. Abb. 7).

Im Zeremonienbuch für ein Blasinstrument (196o-1982) ignoriert Schneider ostentativ die damaligen Haupttrends der zeitgenössischen Musik. Gerade bei der Musik für Blasinstrumente wurden in den 196oer- und 197oer-Jahren viele Spezialeffekte und Verfremdungen verwendet. Heinz Holliger komponierte beispielsweise zur gleichen Zeit seine extremen Werke für die Oboe (Cardiophonie und Studie über Mehrklänge) und auf vielen Schreibtischen der

12 Alle Namen und Datierungen aus Schneider: Zeremonienbuch, S. 1 (Partitur). Weil Schneider im Liederbuch und im Zeremonienbuch alle Datierung mit der Ziffer 5 enden lassen will, schreibt er bei Robert Walsers Und ging »ca. 1895«. Dieses ist wie das Gedicht Beiseit (vgl. Anm. 10) erst im August 1899 in der Wiener Rundschau erschienen. Weil Schneider für Chorbuch und Orchesterbuch Texte aus Die Rose aus dem Jahr 1925 verwendet, kann er die Regel der Ziffer 5 als Endzahl der Datierungen auf alle Vier Bücher ausdehnen.

13 Auch in späteren Werken von Urs Peter Schneider taucht Ludger von Diedrichsfeld auf, unter anderem in Zusammenhang mit einer ebenfalls nicht nachweisbaren »Margot Erdmute von Drajschenfleygh«, wohl ein Pseudonym für seine zweite Frau, Marion Leyh. In seiner Publikation Urs Peter Schneider. Komponieren 1955 bis 1988 ist Ludger von Diedrichsfeld Redaktor der Texte, des Werkverzeichnisses sowie Verfasser der Biografie. Fraglich ist auch die Existenz von Sänger von Treuchtlingen. 


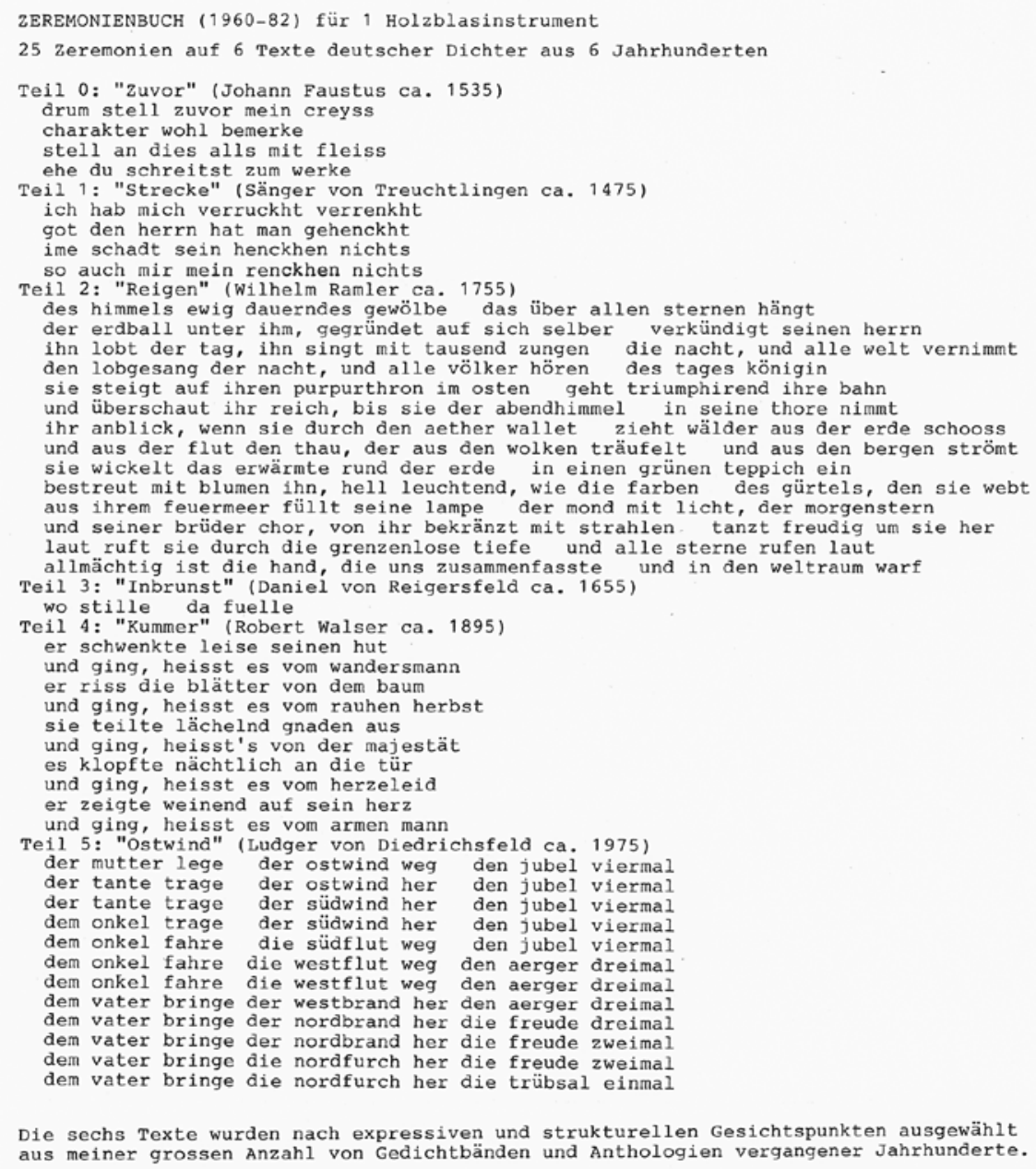

Abb. 7 Urs Peter Schneider: Texte zu Zeremonienbuch (196o-1982)

Komponisten stand ein wichtiges Rezeptbuch, der sogenannte $>$ Bartolozzi $<,{ }^{14}$ eine Sammlung vor allem von Mehrklängen, die man mit speziellen Griffen und Anblastechniken bei Blasinstrumenten realisieren kann. Bei Schneider findet sich davon nichts. Er hat scheinbar einfache, modellhafte Melodien komponiert - die es jedoch in sich haben.

In »Teil IV«, dem fünften Teil des Zeremonienbuchs, der Robert Walser gewidmet und mit Kummer überschrieben ist, wird die musikalische Lakonie 
auf die Spitze getrieben. Es handelt sich um fünf instrumentale Versionen des Gedichts Und ging (SW 13, 27). Schneider nennt es in der Partitur: »Fünf langsame Gänge durch melancholische Bezirke ... Auslaufen und Verschwinden, Lücken und Schweigen ...« ${ }^{15}$

Walsers Gedicht besteht aus zehn Zeilen, die als fünf Doppelverse gestaltet sind. Jeder Vers hat acht Silben, das ganze Gedicht also 8o Silben. Diese Zahlen - fünf, acht und zehn - strukturieren die fünf Stücke. Mit Ausnahme des letzten weisen alle kurzen Studien aus Kummer 8o Töne auf.

Jede Studie akzentuiert einen anderen Aspekt des Textes: Kummer 1 und Kummer 5 thematisieren die komplexen periodischen Überlagerungen in Walsers Gedichten. Kummer 2 zeigt die schlafwandlerische Sicherheit und zugleich die Präzision von Walsers Schreiben, indem über einem nicht hörbaren Raster von 200 Achteln, die sich in acht Abschnitte von fünf mal fünf gliedern $(8 \times 5 \times 5=200)$, acht freie Melodien von je zehn Tönen gebildet werden. Kummer 4 zeigt ein absinkendes Aufsteigen oder ein aufsteigendes Absinken, indem fünf Melodien von je 16 Tönen in Skalenfragmenten aufsteigen, als Ganzes aber absteigen, sodass zum Schluss nur knapp eine Oktave ausgeschritten wird. Kummer 3 bildet schließlich das Zentrum und das Herz dieser fünf Walser-Stücke (vgl. Abb. 8). Schneider schreibt in der Spielanweisung:

Utopischer Choral. Jeweils Legato bis zur Zäsur, und diese lange beziehungsweise, bei Fermate, sehr lange. Kreuz hoch und scharf, Be tief und stumpf intonieren. Fünf Typen von Vibrato, 1 senza, 2 schnell und flach, 3 poco, 4 langsam und weit, 5 molto. Es soll versucht werden, hohe Töne eher eilig und leis, tiefe Töne eher ruhig und laut zu spielen. Jedenfalls sollen die einzelnen Verse rhythmisch instabil, dynamisch inegal erklingen, doch nicht zu extrem. Vorzeichen gelten nur für eine Note. ${ }^{16}$

Das Vibrato wechselt bei jedem Ton, und weil das Vibrato das wichtigste expressive Mittel in der westlichen Musik geworden ist, changiert auch die Emotionalität der Musik bei jedem Ton, wobei - Ordnung muss bei Schneider immer sein - jede der fünf Vibratostufen 16 -mal vorkommt $(5 \times 16=80)$. Durch die leichten Verstimmungen, die mit den geschärften Kreuzen und den vertieften Bes entstehen, ergibt sich nicht nur ein großer Reichtum der Klangfarben, sondern auch der Intervalle: Es wird ein enharmonisches Hören mit Anklängen an Tonales evoziert, das sich aber doch nie einlöst.

\footnotetext{
15 Schneider: Zeremonienbuch, S. 34.

16 Ebd.
} 


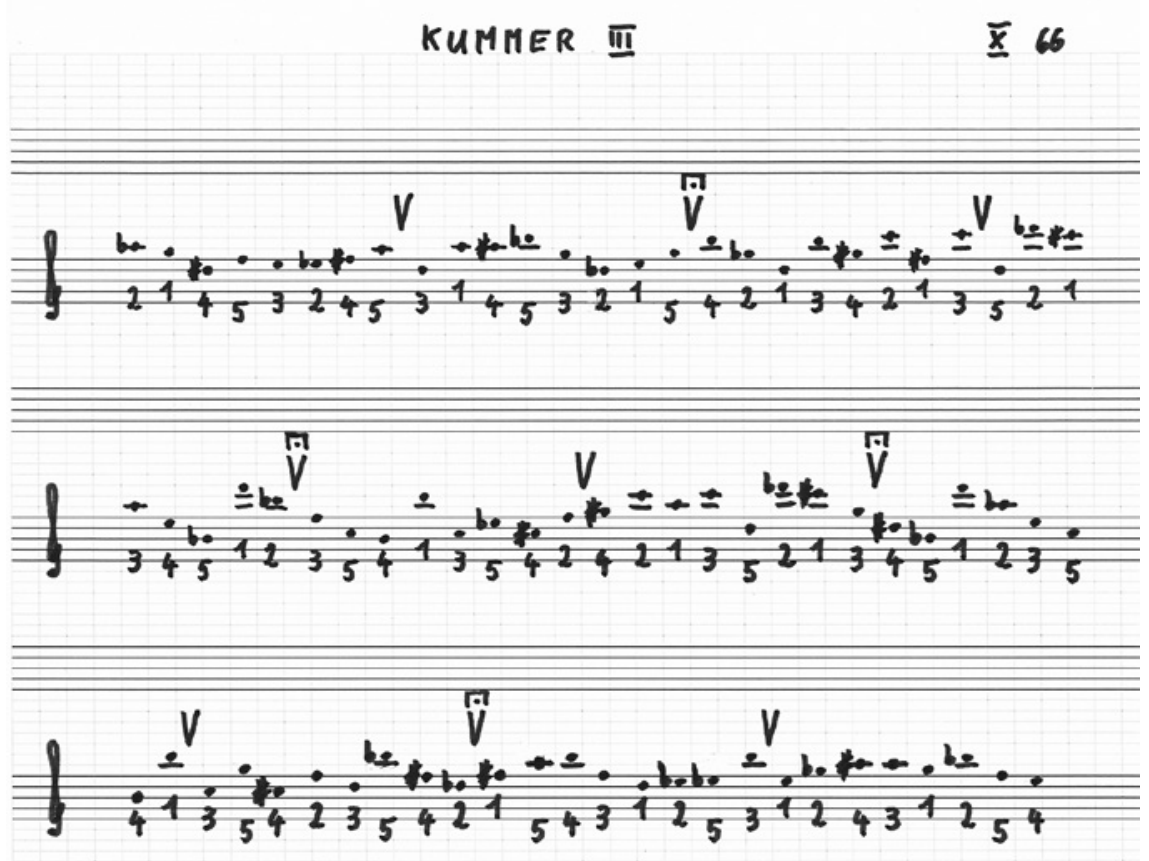

Abb. 8 Urs Peter Schneider: Kummer 3 aus Zeremonienbuch (196o-1982)

\subsection{Die reinen Walser-Bücher}

Das Chorbuch und das Orchesterbuch ${ }^{17}$ sind ausschließlich mit Texten von Robert Walser komponiert. Sie entstammen durchwegs Walsers letzter von ihm selbst betreuten Publikation Die Rose, 1925 bei Rowohlt erschienen. Schneider wählt jeweils einzelne Sätze aus und komponiert darüber je eine »Zeremonie«, wie er auch hier die Stücke nennt. Innerhalb dieser Sätze entscheidet er sich meist für ein Schlüsselwort, das dem Stück auch den Titel gibt, zum Beispiel im Orchesterbuch der Teil Fabrik. Walsers Satz lautet: »Eine Fabrik zur Gewöhnlichmachung des Ungewöhnlichen scheint im Gange«. Schneider bemerkt dazu:

17 Chorbuch (1966-1977): 12 Lieder auf 12 Texte von Robert Walser (1925) für 8 Singstimmen. Orchesterbuch (1974-1981): 8 Zeremonien auf 8 Texte von Robert Walser (1925) für 8 Holzbläser und Streicher. 
Blick des Betrachters auf plakative Lebensweisen, auf Schrumpfung des Seltsamen und Einstampfung des Bedeutenden ... Im grauen Taktschritt ... Zum Kult der Langeweile zwei langweilige Beispiele ... ${ }^{18}$

Schon das Partiturbild (vgl. Abb. 9) zeigt quasi die `Fabrik«, bei der die Stimmen wie Zahnrädchen ineinandergreifen und jede der anderen folgt. Jede Stimme beruht auf einer >Fünfzehntonreiheく, die - analog zur Zwölftontechnik - alle Töne innerhalb einer None verwendet. Anhand der ersten Stimme (oberstes System) sei das Vorgehen demonstriert:

Die eine große None umfassende Fünfzehntonreihe besteht aus den folgenden 15 Tonhöhen:

$$
c^{2}-f i s^{2}-g^{2}-c i s^{2}-g i s^{1}-g^{1}-h^{1}-f^{2}-a^{2}-g i s^{2}-d^{2}-a^{1}-b^{1}-e^{2}-e s^{2}
$$

Wenn man die Reihe dodekaphon betrachtet, könnte man auch sagen, es handelt sich um eine Zwölftonreihe, bei der drei Tonhöhen oktavverschoben verdoppelt werden. Diese drei Tonhöhen (fett gesetzt) sind - auch in den Intervallkonstellationen - symmetrisch in die Fünfzehntonreihe eingepasst. Mit Wiederholungen, die in der akademischen Dodekaphonie >erlaubt sind, erweitert Schneider die Melodie auf 26 Töne (vgl. Abb. 9).

Fasst man zusammen, wie sich die Repetitionen auf die fünfzehn Tonhöhen der None $g^{1}-a^{2}$ verteilen, ergibt sich erneut eine streng symmetrische Konstellation um die Mitte des vierfachen $d^{2}$, das mit den dreimal repetierten Tönen $f i s^{2}$ und $b^{1}$ (fett gesetzt) einen übermäßigen Dreiklang bildet:

$\begin{array}{ll}g^{1} & 1 \\ g i s^{1}-g i s^{1} & 2 \\ a^{1} & 1 \\ \boldsymbol{b}^{1}-b^{1}-b^{1} & 3 \\ h^{1} & 1 \\ c^{2}-c^{2} & 2 \\ c i s^{2} & 1 \\ d^{2}-d^{2}-d^{2}-d^{2} & 4 \\ e s^{2} & 1 \\ e^{2}-e^{2} & 2 \\ f^{2} & 1 \\ f i s^{2}-f i s^{2}-f i s^{2} & 3 \\ g^{2} & 1 \\ g i s^{2}-g i s^{2} & 2 \\ a^{2} & 1\end{array}$

Auch hier ist viel struktureller Humor gegenüber seinen Zeitgenossen aus der Zwölftonschule zu beobachten. Einerseits limitiert Schneider die verwendeten Intervalle auf insgesamt vier: fünf Tritoni, zwei Quarten, zwei große Terzen 
FABRIK I

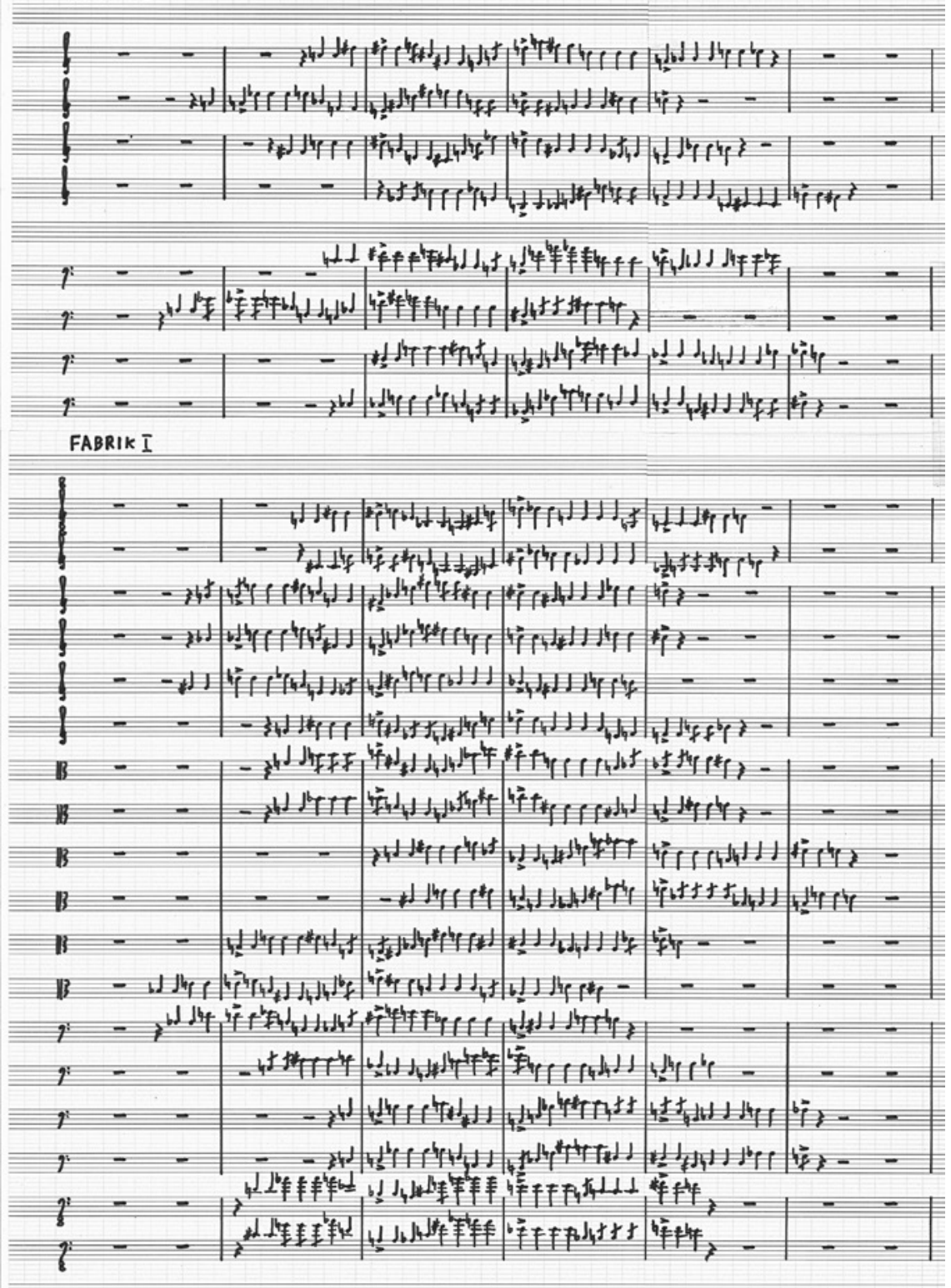

Abb. 9

Urs Peter Scheider: Fabrik aus Orchesterbuch (1974-1981)

und fünf kleine Sekunden. Damit ahmt er verblüffend den akademischen Zwölftonstil nach. Andererseits legt er mit den Repetitionen den Finger auf einen wunden Punkt der Zwölftontechnik: Die Regel, dass ein einmal erklungener Ton nicht wiederholt werden darf, bevor alle anderen erklungen 
sind, der Ton selber aber unbeschränkt repetiert werden kann, gibt zwar >Freiheiten < bei der Suche nach spannenden harmonischen Konstellationen, entbehrt aber strukturellen Sinns. Denn das ästhetische Ziel der Dodekaphonie, alle Töne gleichwertig zu behandeln, wird mit der Repetitionsregel konterkariert, weil kaum ein anderes musikalisches Stilmittel sich rhetorisch so in den Vordergrund drängt wie die Wiederholung.

Schneider zwingt diese Repetitionen, die in der akademischen Dodekaphonie oft zu signalähnlich klopfenden Motiven führen, in ein symmetrischmaschinelles Raster, das alle Einzelstimmen der Fabrik in jeweils wechselnder Permutation bestimmt. In den Spielanweisungen verlangt Schneider $»[\mathrm{~m}]$ öglichst zwölftönig temperierte Intonation, also zum Beispiel as gleich gis «, ${ }^{19}$ sodass das typische Grau in Grau akademischer Zwölftonwerke hörbar wird. Schneider komponiert also quasi eine >Zwölftonfabrik<, bei der 26 Stimmen während 40 Vierteln nacheinander 26 Töne spielen. Die Fabrik und die normierte Zwölftonintervallik erscheinen so als Walser'sche Gegenwelt, zugleich aber auch als in die Struktur eingeschriebene Kritik der Zwölftonorientiertheit zahlreicher Zeitgenossen von Schneider. In einer Randnotiz schreibt er auf einer Kompositionsskizze zu Fabrik I: »Der Dirigent dirigiert, bevor und nachdem die Instrumente agieren - >der Chef (der Fabrik) kommt als erster und geht als letzter (Schneider-Witz) «. ${ }^{20}$

Auch das Chorbuch basiert durchwegs auf Auszügen aus Die Rose, die Schneider in ähnlicher Technik in Musik setzt, oft mit >Polyphonisierung< des gleichen Textmaterials, manchmal des gleichen Wortes. Gewisse Lieder sind von bestechender Einfachheit, ja provozierender Simplizität, zum Beispiel Sinken (vgl. Abb. 10). Walsers lakonischen Satz »Sinken kann vorteilhafter sein als Steigen « (SW 8, 73) vertont Schneider in einer zweistimmig absinkenden Skala. Plakativer geht es nicht - aber auch hier beachte man den >strukturellen Humor: Nicht nur, dass die Skala auf die zweimal vier Stimmen verteilt und durch abwechselnden 4/8- und 3/8-Takt belebt ist, auch die Skala selbst hat es in sich, sie wirkt diatonisch, ist aber eine Achtton-Skala, die dadurch entsteht, dass sich Ganzton- und Halbtonschritte regelmäßig abwechseln. Grundsätzlich kann man von einem beliebigen Ton aus nur zwei solche Skalen bilden, nämlich eine, die mit einem Halbtonschritt beginnt, und eine andere, die mit dem Ganztonschritt beginnt. Mit diesen beiden Achttonskalen realisiert Schneider die Zweistimmigkeit von Sinken: Die Soprane beginnen ihren Abstieg mit dem Halbton, die Bässe mit dem Ganzton. Dasselbe ist nicht dasselbe und ergibt

19 Schneider: Orchesterbuch, S. 27.

20 Privatarchiv des Komponisten. 


\section{SINKEN I}

SINKEN II

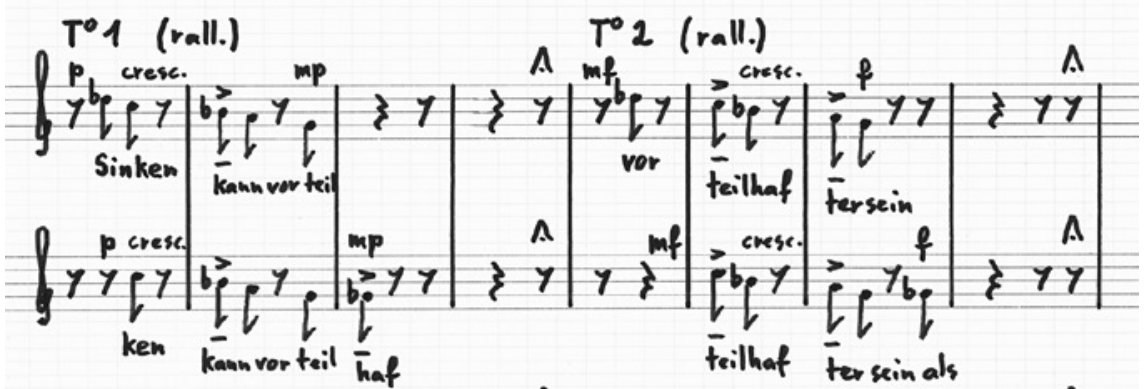

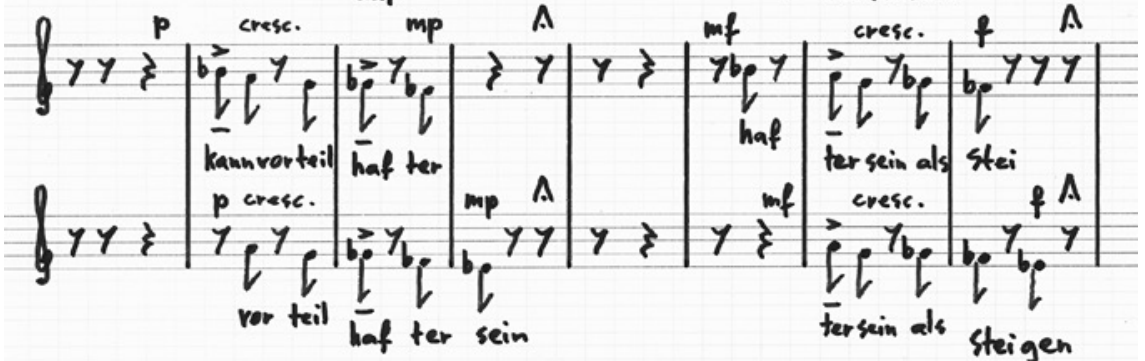

SINKEN I

SINKEN II

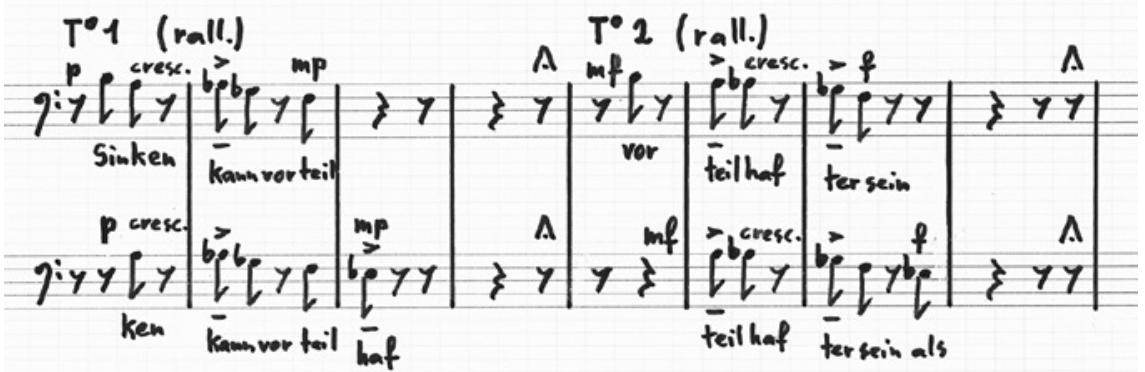

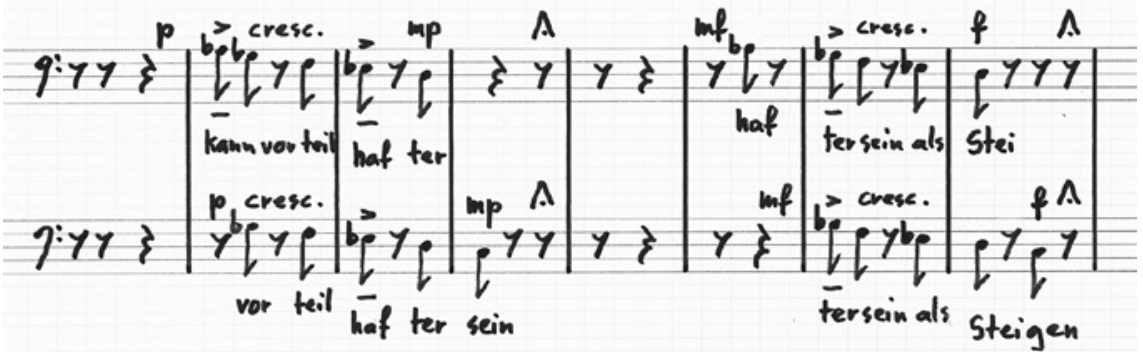

Abb. $10 \quad$ Urs Peter Schneider: Sinken aus Chorbuch (1966-1977) 
bei diesem Sinken der Skala eine irisierend-irritierende Farbigkeit, deren Komplexität kaum ein Hörer aufschlüsseln kann. Dazu kommt noch etwas Weiteres: Die absteigende Skala erreicht den sie einlösenden Oktavton nicht, sondern bricht auf dem achten Ton ab, was die Skalen in »verblüffte Stillen münden « lässt, wie Urs Peter Schneider in den Spielanweisungen notiert. ${ }^{21}$

Schneiders Walser-Auseinandersetzungen in Die vier Bücher erinnern an allegorische Embleme und Sinnbilder, die mehrdeutige und vielschichtige Bezüge aufweisen und einer intensiven Aufschlüsselung bedürfen, wenn man sie verstehen will.

\subsection{Spazieren mit Robert Walser}

1975/76 bekam Urs Peter Schneider auf Einladung des damaligen Musikchefs von Studio Bern des Radios der deutschen und rätoromanischen Schweiz die Möglichkeit, ein >radiophonisches Porträt< unter dem Titel Spazieren mit Robert Walser zu realisieren. Die Anfrage kam zu einem speziellen Moment der Radiogeschichte, denn man begann sich damals auf die Stereotechnik vorzubereiten, die ab 1978 schrittweise auf dem zweiten Programm und 1986 dann auf allen Kanälen eingeführt wurde. Im einem strengen Plan folgenden Hörstück spielt Schneider mit extremer Stereophonie, wie sie für die frühen stereophonischen Hörspielarbeiten typisch ist: »Landschaften und Begebenheiten ziehen, als wären die Objekte verschieden weit vom Spazierenden entfernt, am Hörer vorbei. $\ll^{22}$ Die Schneider so wichtige >verzerrte Symmetrie ist auch hier wieder zu hören, nämlich als »beschwingter Hinweg und dämonisierter Rückweg «. ${ }^{23}$ Schneider schreibt dazu:

»Symmetrien« sind bei mir: a) Zentralsymmetrien aller Parameter, b) »Umstülpungen« ab einer Mitte ins Gegensätzliche, c) Mehrere gegeneinander verschobene, nach dem Beispiel Weberns, d) Nie (!) zeitlich(e) simple Rückläufe. ${ }^{24}$

Dominierend sind aber die Walser-Sätze, die - ruhig und fast zeremoniell wie eine Pendeluhr - im Minutentakt vorgetragen werden, und zwar von einer der damals bekanntesten Stimmen, jener von Susanna Enz, die während Jahrzehnten die Nachrichten des Schweizer Radios möglichst objektiv und

\footnotetext{
21 Schneider: Chorbuch, S. 7.

22 Schneider: [Einführung ins Hörstück], Typoskript, Privatarchiv des Komponisten.

23 Ebd.

24 Brief und Material von Urs Peter Schneider an Roman Brotbeck vom 04.09.2016.
} 
emotionslos gelesen hatte. Auch die Texte von Walser liest sie wie Nachrichten und vermeidet jegliche Gestaltung. Die kurzen Walser-Texte bilden nämlich nur eine von fünf Ebenen dieses polyphonen Hörstücks, dessen Mitte Urs Peter Schneider mit einem berndeutsch gesprochenen Kommentar markiert: »Aber doch so läng, dassme auso mitem Vergässe cha spile. « ${ }^{25}$ Auf den anderen Ebenen erklingen klar erkennbare Objekte wie Schritte, Schlagzeug, Sopran, Kinderchor, Flöte und andere, aber alles ergänzt sich zu einem 34 Minuten dauernden Walser-Panoptikum und wirkt wie eine langsame und stets ruhige Introspektion von Robert Walser. Auch im Medium Radio dominiert wiederum, was Schneider als seinem Werk Gemeinsames schon betont hat: »Non-Espressivo, Non-Hysterie, Nicht-Dramatik «. ${ }^{26}$

\subsection{Verschneiderter Walser: Die Trilogien}

Die Arbeit an den Vier Büchern schloss Urs Peter Schneider 1981 mit dem achtzigsten Stück Inhalt ab. Dieses bezieht sich auf Robert Schumann und ergibt eine interessante Parallele zum acht Jahre später entstandenen Beiseit-Zyklus von Heinz Holliger, bei dem sich der letzte Gesang ebenfalls auf Schumann bezieht. Die Grundlage für das Stück von Schneider bildet ein Walser-Satz, der sich direkt mit dem >verrückt< gewordenen und aus allen Positionen gejagten Robert Schumann in Düsseldorf verbinden lässt: $\gg \mathrm{Zu}$ einem Verunglückten aus übereinandergebrochener Empfindung sagt die Mitwelt nein «. (SW 8, 71) Schneider verwendete dafür Schumanns letzte Komposition, die Geistervariationen, deren Thema gemäß Schumanns Frau Clara von den Geistern Schuberts und Mendelssohns dargebracht wurde (vgl. Kap. 8.6.3). Allerdings ist Schneiders Bearbeitung des Themas so radikal, dass ein nach melodischen Reminiszenzen suchendes Ohr vorerst, im ersten der zwei Teile, nichts von Schumann hören wird, denn die Tonhöhen werden zu Tondauern und umgekehrt. Der Klang allerdings erinnert an jene Kälte, die viele WalserVertoner gerade am Schluss ihrer Werke suchen.

Nur sechs Jahre später, 1987, beginnt sich Urs Peter Schneider erneut mit Walser auseinanderzusetzen. Und auch diesmal wird es eine Beschäftigung, die sich über insgesamt 17 Jahre hinzieht. Sie umfasst drei Trilogien, total neun Kompositionen, die ihrerseits in einen noch größeren Zyklus eingebunden sind, denn sie bilden das Bindeglied zwischen den drei Hölderlin- und den drei Sauschneider-Trilogien, also das Zentrum eines Gesamtkonzepts von

25 »Aber doch so lange, dass man mit dem Vergessen spielen kann.«

26 Brief von Urs Peter Schneider an Roman Brotbeck vom 08.07.2016. 
27 Kompositionen bzw. drei mal drei mal drei Stücken. Als würde Urs Peter Schneider auch seine Biografie und sein Schaffen nach Zahlen ausrichten, ergeben sich zwischen den einzelnen Walser-Trilogien jeweils Pausen von sieben Jahren. Der erste dieser Unterbrüche war ein tatsächlicher Kompositionsunterbruch, denn nach 1988 legte Urs Peter Schneider das Komponieren auf Eis und verlegte sich für mehrere Jahre aufs Schreiben von Texten, Kommentaren und literarischen Werken. Die drei Walser-Trilogien setzen sich wie folgt zusammen:

Robert Walser Trilogie I (1987/88)

1. Die schöne Frau von Thun (1987)

Sechs Lebensbilder

für drei Paare von (Streich- und/oder Blas-)Instrumenten

Susanne Schöni zugedacht

2. Tobold (1987/88)

Szenen der Demut

für Chorstimmen und Orchestergruppen

für Christine Volkmann

3. Das Mädchen mit den schönen Augen (1988)

Zwei Albumblätter

für Oboe und Trompete (oder zwei andere, aber leicht verschiedene Instrumente)

Marion Leyh zugedacht

Robert Walser Trilogie II (1995/96)

1. Studje zwej (1995)

für Sprecher oder Saxofon solo (et al)

für Bruno Spoerri zum 6o. Geburtstag

2. Studien über den Adel (1995/96)

für vier Ausführende (Multimediales Werk)

für Pierre Sublet

3. Studje drej (1996)

für Sprecherin oder Harfe solo (et al)

für Hermann Meier zum 9o. Geburtstag

Robert Walser Trilogie III (2003/04)

1. Die Liebe in den leichten Worten (2003)

Zwei Albumblätter für Fiedel und Gambe (oder zwei andere, aber leicht verschiedene Instrumente)

Renate Heydel zugedacht

2. Kanal (2003/04)

Szenen der Anmut

für Sprechstimme und Kammerensemble

für Babette Schweizer

3. Das leichte End in Bern (2004)

Sechs Lebensbilder für drei Paare von (Zupf- und/oder Schlag-)Instrumenten

Eva Fuhrer zugedacht 
Noch stärker als die Vier Bücher sind die Walser-Trilogien von vielen Symmetrien und kompositorischen Bezügen geprägt, die sich um die Mittelachse von Studien über den Ade ${ }^{27}$ (II, 2) spiegeln. Das geht bis in die Titellänge hinein: Das erste (I, 1) und das letzte Stück (III, 3) enthalten im Titel sechs Silben und beziehen sich auf die beiden Städte Thun und Bern; die Anzahl Buchstaben der Titel differieren nur um einen Buchstaben (2o bzw. 19 Buchstaben); das dritte (I, 3) und das drittletzte Stück (III, 1) haben im Titel dieselbe Differenz (28 bzw. 27 Buchstaben) und auch die gleiche Anzahl Silben (9) bzw. Worte (6). Wer hier sucht, wird zahlreiche weitere Fast-Symmetrien finden, zum Beispiel in der Besetzung, in der Behandlung der Stimme und sogar bei den Widmungen. Die mittlere Trilogie ist nur Männern zugedacht - wobei zwei Komponisten einen Interpreten umklammern -, die erste und letzte Trilogie nur Frauen, wobei sich sogar die Art der Widmungen symmetrisch zueinander verhalten (»für« und »zugedacht« wechseln sich ab).

Die Walser-Trilogien unterscheiden sich ganz grundsätzlich von den Vier Büchern, denn Schneider >übersetzt< hier die Walser'schen Texte nicht in Musik, um Allegorien zu gestalten, sondern er verwendet die Texte selber als musikalisches Material. Als »Transformationen« bezeichnet es Schneider selbst:

Die auf Walser bezogenen 9 Stücke beschäftigen sich (fast durchwegs) mit Transformationen Walserscher Texte (in neue Texte, in musikalische Parameter, in musikalisch-semantische Topoi). ${ }^{28}$

Dieses Verfahren ist in der musikalischen Walser-Rezeption einmalig. ${ }^{29}$ Schneider sucht bei jedem Stück eine andere Form der Texttransformation. Allen Vorgängen gemeinsam ist, dass die Semantik von Walsers Originaltext verschwindet; auch der beste Walser-Kenner wird die von Schneider transformierten Texte nicht >verstehen<, und ohne Kenntnis von Schneiders Transformationstabellen sind sie auch nicht rückübersetzbar. Trotzdem bezieht sich alles bis ins Detail auf Walsers Original. Die Verfahren erinnern an die literarischen Experimente der Literaten- und Mathematiker-Gruppe Oulipo ${ }^{30}$ oder an Kurt Schwitters, der in vergleichbarer Weise Buchstaben, Silben und

27 Der Zwischentitel in Robert Walsers Tobold (II) lautet Studie über den Adel (BA 13, 96-123, hier 107); der Kompositionstitel von Schneider trägt den Plural Studien über den Adel.

28 Brief von Urs Peter Schneider an Roman Brotbeck vom 04.09.2016.

29 Im Bereich der bildenden Künste nimmt der französische Künstler Frédéric Diart ( ${ }^{*} 1966$ ) eine vergleichbare Position ein. Er verarbeitet in seinen visuellen Arbeiten zu Robert Walser ausschließlich Buchstaben und Texte, die in den gestalterischen Prozessen oft fast vollständig verschwinden. Vgl. Scrawitch: Frédéric Diart.

Vgl. Bisenius-Penin/Petitjean: 50 ans d'Oulipo. 
ganze Wörter wie Töne behandelt hat. Schneider hat auch als Schriftsteller, ohne musikalische Komposition, solche Texttransformationen erstellt. ${ }^{31}$

Das mittlere Stück der neun Kompositionen - und auch des gesamten 27-teiligen Trilogien-Komplexes - zeigt einige besonders vielschichtige Überlagerungs-, Übersetzungs- und Transformationsprozesse, weil Schneider hier über die Musik hinausgreift, um ein multimediales Werk zu konzipieren.

Dass ich genau in der Mitte der 27 Stücke »Studien über den Adel« (ein WalserTitel) theatralisch umsetze, ist einem Rückblick auf meine frühen theatralen, multimedialen, szenischen Arbeiten geschuldet, die lange (als noch niemand so komponierte in der Schweiz, ausser [Hans] Wüthrich) [...] einen »Bauchnabel« meines Tuns repräsentierten. ${ }^{32}$

Wie schon bei den frühen szenischen Arbeiten von Schneider kann man auch hier nicht von sinstrumentalem Theater in der Tradition von Mauricio Kagel oder von Georges Aperghis' Théâtre musical sprechen. Es fehlen die Zuspitzungen, die sich in einem unerwarteten Schock oder in einem witzigen Gag entladen; die erzählende Linearität und Einfachheit, deren es zur Vorbereitung solcher Unterbrüche bedürfte, ist nicht Schneiders Welt. Vielmehr läuft bei Studien über den Adel ein ruhiges Ritual ab, bei dem ein Klavierspieler, eine Sprecherin, eine »Hellraumprojektorin« und ein Schreiber in stetem Wechsel agieren und - oberflächlich betrachtet - sechzehnmal dasselbe abläuft: Einem kurzen Klavierstück folgt eine Lektüre in deutscher Lautung, aber mit unverständlichem Inhalt, dann wird ein Hellraumprojektor angeknipst, der sternförmig überlagerte Partituren auf eine Leinwand wirft, schließlich stellt sich ein Schreiber mit dem Rücken zum Publikum zwischen den Hellraumprojektor und die Leinwand und imitiert vergrößerte Schreibbewegungen. Die Folien sind so konstruiert, dass man sie wie eine Sternkarte drehen kann, was die »Hellraumprojektorin « - wie Schneider die Frau am Hellraumprojektor nennt - dann auch tut, wobei sie bei jedem Durchgang eine der sechzehn Folien wegnimmt, bis zum Schluss nur noch weißes Licht projiziert wird. Alle Personen sind schwarz gekleidet, mit Ausnahme des in weißem Kleid agierenden Schreibers. Nach einem »sanften Lichtlöschen« folgt die nächste Variation dieses Ablaufs. »Eine Aufführung hat den zeremoniell sich abspiegelnden Haltungen, dem Sich-Wundern-Können, Rechnung zu tragen. $^{33}$ Dieses Zeremonielle wird mit Kerzenbeleuchtung bzw. schwachem Scheinwerferlicht zusätzlich betont.

31 Schneider: Schriften I bis V 1955-2015. Die Schriften sind ein Buchkonzept ohne Seitenzahlen und in sich symmetrisch angelegt.

32 Brief von Urs Peter Schneider an Roman Brotbeck vom 04.o9.2016.

33 Schneider: Walser-Trilogie II, 2: Studien über den Adel, S. [o]. 
Die textliche Grundlage der Komposition ist ein minimal veränderter Ausschnitt aus Walsers Studie über den Adel:

Wie ich mich erinnere, schrieb ich einmal folgende geheimnisvolle Studie über den Adel und sandte diese knappe und kurze Abhandlung warm und eilig an die Redaktion eines bedeutenden Tageblattes; die Bemühung erwies sich jedoch als nutzlos, das Geistesprodukt blieb ungedruckt und wanderte wahrscheinlich in den jederlei derartige verschwendete Anstrengungen aufschnappenden Papierkorb, was der Autor natürlich innig bedauerte, ohne aber zornig zu werden, da er nie ein großer Schriftsteller sein zu sollen meinte. ${ }^{34}$

Diesen Text übersetzt Schneider zunächst in ein symmetrisches phonetisches Alphabet, das es ihm erlaubt, die Laute wie Töne zu verwenden und wie musikalische Motive zu transformieren. Den ins Phonetische übertragenen Satz von Walser unterteilt Schneider in 3 mal 6 Abschnitte von vier Wörtern, also insgesamt 72 Wörter (vgl. Abb. 11) und transformiert das lautlich vereinfachte 〉Original mittels Lautwechseln in sechs verschiedene Varianten, wobei bei diesem >Buchstabenhäckseln` gewisse Wörter den semantischen Sinn noch verfremdet transportieren, während andere reine Lautdichtung werden. So wird das Wort »sriftsteler« im untersten System der Skizze in »hraskhkolor, fruhpfpilir, sreftstalar, sruftstilir, hreskhkalar, frohpfpulur« übertragen.

Aus diesem Transformationsschema wiederum filtert Schneider vier $>$ Gedichte< heraus (vgl. Abb. 12); diese entstehen, indem jeweils die ersten, zweiten, dritten oder letzten Wörter der Vierwort-Gruppen aus den sechs Varianten der Transformationen ausgewählt werden. ${ }^{35}$

Diese vier >Gedichte $<$ - aufgeteilt in vier Strophen à sechs, fünf, vier und drei Zeilen - bilden die Basis für die sechzehnteilige Komposition. Im zwölften Teil der Komposition (vgl. Abb. 13) erscheint in der gesprochenen Partie der »Schriftsteller« bzw. »s'ref't-s'ta-lar«. Das ganze >Gedicht« stellt hier am Ende die Transformation der Wörter »was«, »innig«, »zornig«, »er«, »Schriftsteller« und »meinte« aus Walsers Originaltext dar. Die gesprochenen Teile sollen übrigens nicht mit großem Pathos vorgetragen werden, sondern »etwas beiläufig, nicht theatralisch, mit natürlichem Ausdruck« ${ }^{36}$

34 Zitiert nach den Skizzen des Komponisten. Veränderungen von Schneider kursiv angezeigt. Im Original: »Wie ich mich erinnere, schrieb ich eines Abends folgende geheimnisvolle Studie über den Adel. [...] Diese knappe und kurze Abhandlung sandte ich warm und eilig [etc. wie oben].«(BA 13, 107 und 109)

35 Aus diesen sprachlichen Transformationen hat Urs Peter Schneider auch literarische Texte gestaltet, vgl. Schneider: Schriften, Schriften II, 2 Nummer 27 und Schriften IV, 1 Nummer 79 .

36 Vgl. die Aufführungshinweise in der Partitur. Schneider: Walser-Trilogie II, 2: Studien über den Adel. 


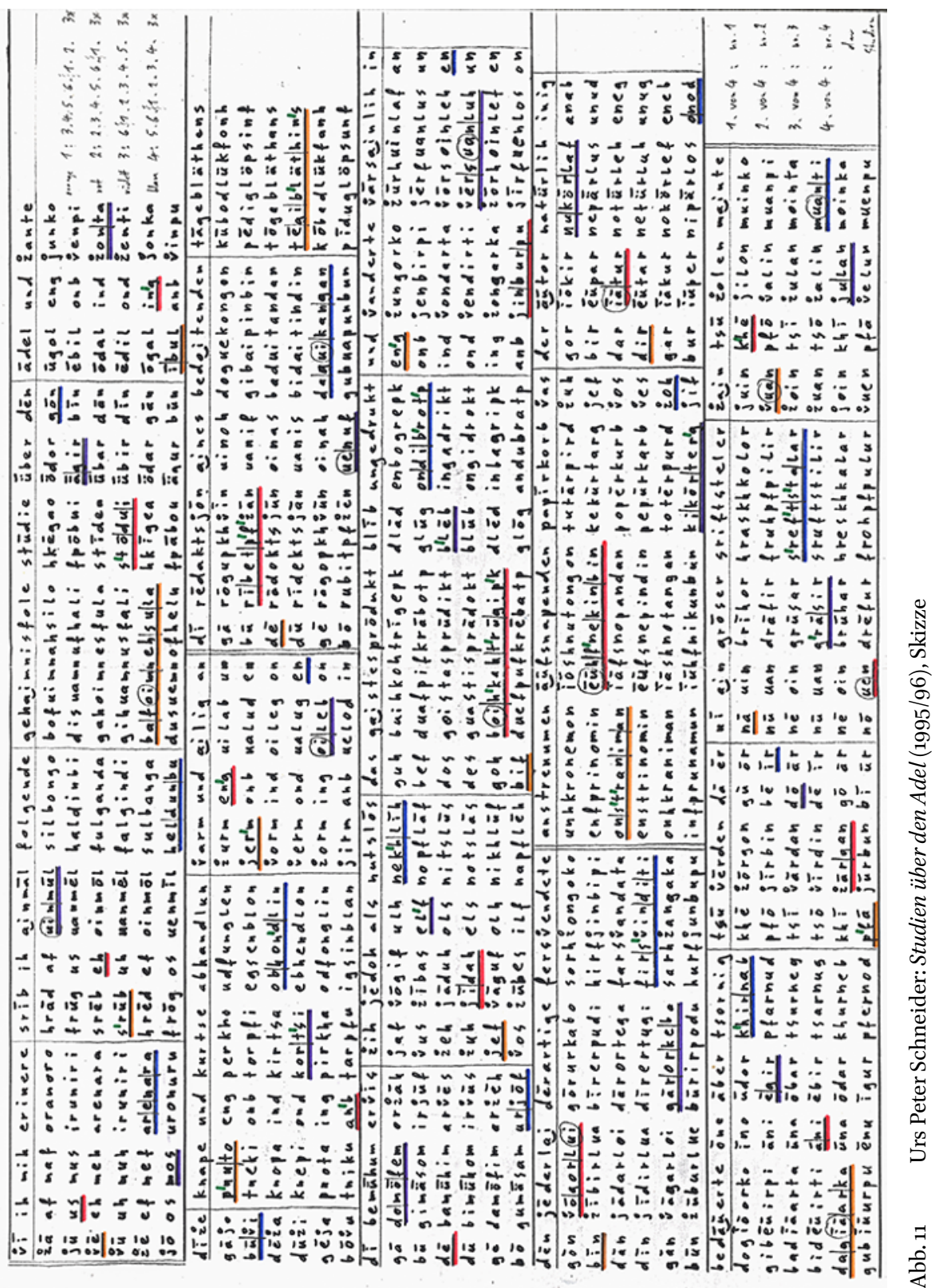




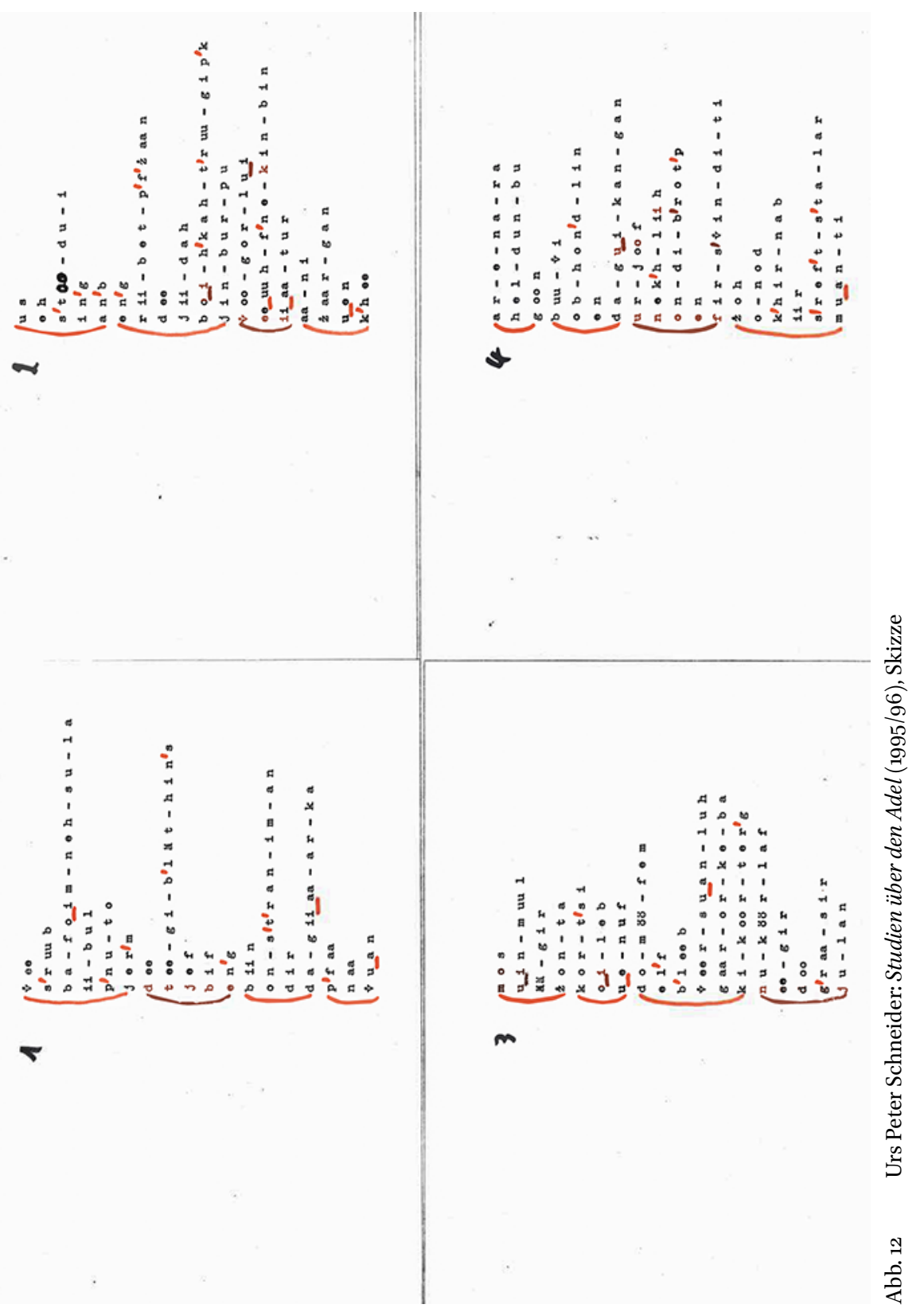




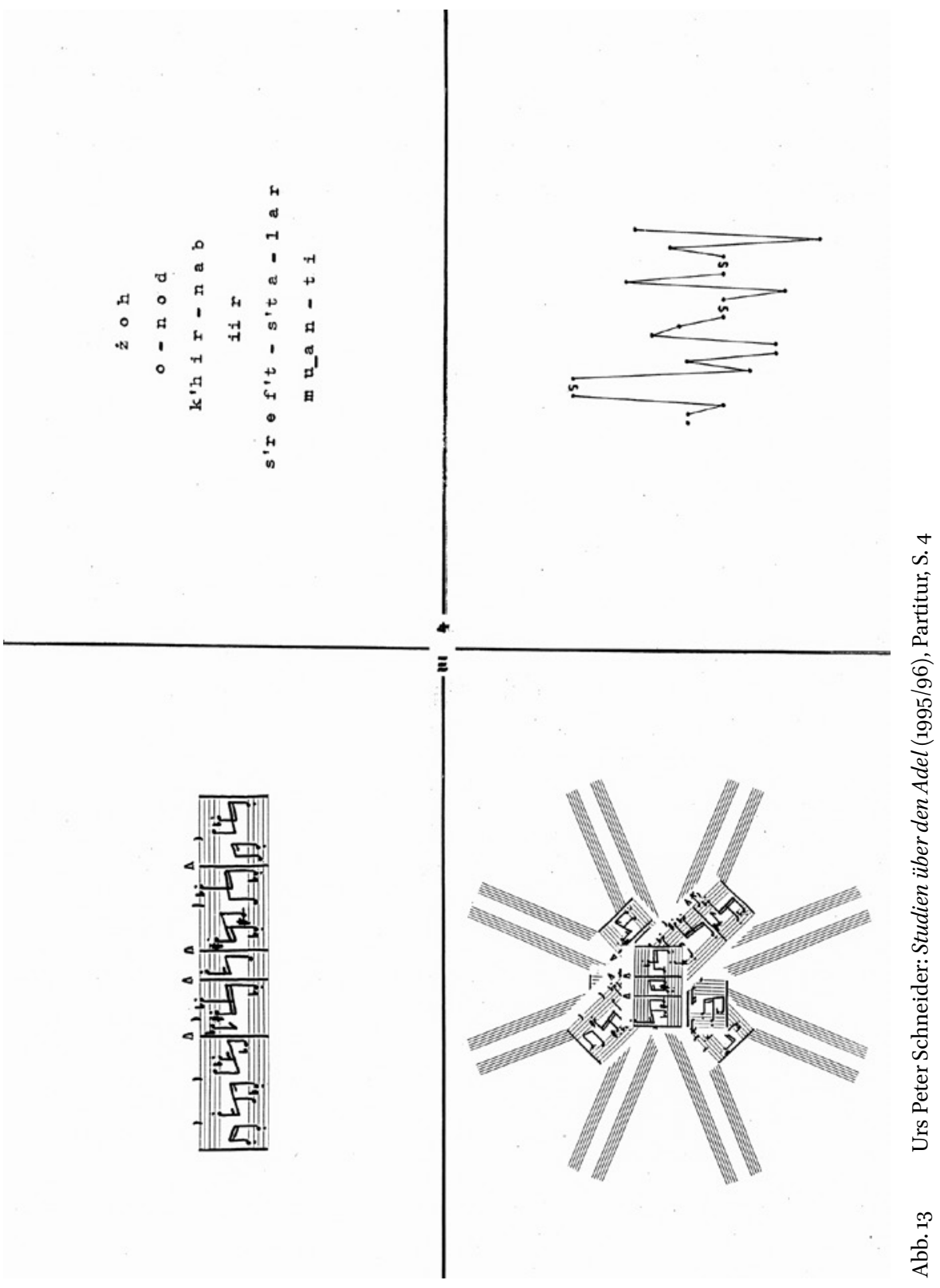


Von diesen Texten aus projiziert Schneider auf die anderen Medien: Der Klavierpart (linke obere Ecke) ist eine Transformation des gesprochenen Textes, die Folien auf dem Hellraumprojektor (linke untere Ecke) eine Transformation der Klaviernoten, die Schneiders zweite Frau Marion Leyh gestaltet hat. Die Bewegungen des Schreibers (rechte untere Ecke) sind erneut vom gesprochenen Text abgeleitet und zeigen nochmals eine andere mediale Spiegelungsform.

Im Gegensatz zu Schneiders Walser-Auseinandersetzungen in den Vier Büchern und in Spazieren mit Robert Walser, die man als >Ver-Walserungen< musikalischer Konzepte bezeichnen könnte, müsste man bei den drei Trilogien umgekehrt von >Ver-Schneiderungen< von Walser-Texten sprechen, denn Schneiders Transformation hat hier die Überhand gewonnen und die mehrfachen Übersetzungen verfremden das Ausgangsmaterial bis zur Unkenntlichkeit. Ohne die Einsicht ins Skizzenmaterial von Urs Peter Schneider wäre es mir unmöglich gewesen, nur schon im Sprachlautlichen einen Bezug zu Walsers Text herzustellen; zu komplex und deshalb irreversibel sind die Transmutationsverfahren.

Bei der Komplexität der vier multimedialen Ebenen kommt dem einzig Konkreten - dem Titel Studien über den Adel - in diesem abstrakten Ritual eine programmatische Bedeutung zu. Wenn man bedenkt, dass in Walsers Text dessen kurze Lebensphase als Diener behandelt wird, entsteht im sinnsuchenden Kopf des Beobachters eine neue Geschichte: Vier Diener arbeiten ihre Zeremonien ab, in immer gleichen Bewegungen und gleichen Rollen, als Hausmusiker der Pianist, als >chef de service $<$ die Sprecherin, als $>$ Fensterputzer der Schreiber, dessen weite Ausschläge vor der Leinwand gleichsam die makroskopische Umkehrung von Walsers Mikrogrammen sein könnten. Und die »Hellraumprojektorin« dreht an ihren Scheiben, als würde sie eine leerlaufende Uhr aufziehen und zunehmend demontieren, bis zum Schluss nur das reine Weiß der leeren Zeit übrigbleibt.

\subsection{Werke für die Zukunft}

Zum 5o. Todestag von Robert Walser komponierte Schneider 2006 sein bisher zweitletztes Werk mit einem Walser-Bezug: Seitab für Celestaklänge. Es ist erneut ein ausgeklügeltes und auf mehrfachen >Übersetzungen< basierendes Konzept für eine bis sechs Celestas, deren Klang einem Glockenspiel ähnelt. Die verblüffende Pointe des Kompositionskonzepts besteht darin, dass mit 
zunehmender Anzahl Celestas tendenziell weniger Töne erklingen. Im alldominierenden Glöckleinklang wird man an eine Himmelfahrt erinnert - auch dies eine Gegenposition zu jener Walser-Welt der Stille, die viele Komponisten entwerfen und die auch Schneider selber beim Schumann-Stück der Vier Bücher suchte.

In der Vollbesetzung mit sechs Celestas wird das Werk nur selten gespielt werden, weil es einen großen Aufwand mit sich bringt, sechs kompatible Exemplare des raren Instruments aufzutreiben. Aber solche utopischen Konzepte sind gerade für die jüngsten Werke Schneiders bezeichnend: Er liebt es, Werke zu konzipieren, die sich heute erst teilweise realisieren lassen, in unzähligen Versionen und Besetzungen aufgeführt werden können oder deren vollständige Uraufführung er selber womöglich gar nicht mehr erleben wird. ${ }^{37}$

Zum 6o. Todestag von Robert Walser schrieb Schneider »zur eigenen Verblüffung ${ }{ }^{38}$ sein wohl letztes Werk über den Schriftsteller, das Klaviertrio Teich mit zwei Schwänen (vgl. Abb. 14). ${ }^{39}$ Obwohl dieses Klaviertrio eine eigene, vom Text des Dramoletts Der Teich (SW 14, 119-132) losgelöste Musik darstellt, kann es für Schneider auch als »Zwischenaktmusik zu einer eventuell nie stattfindenden Aufführung >in Bieler Mundart « gespielt werden. ${ }^{40}$ Der $>$ Miniatur-Shakespeare Theater-Szene (vgl. Kap. 8.5.1 und 8.6.4), den dieses Dramolett darstellt, sollte gemäss dem Komponisten »rein lyrisch, als unaufgeregte, reine Textlesung mit verteilten Stimmen « aufgeführt werden, zu der das Trio zehn Meditationen »über mögliche und unmögliche Septimenakkorde« beisteuert. ${ }^{41}$ - Man möchte mit Urs Peter Schneider insistieren: »Non-Espressivo, Non-Hysterie, Nicht-Dramatik. ${ }^{42}$

37 Ein schönes Beispiel sind Dieselben (2009/10), vier Stücke für die Carrillo-Klaviere (Halb-, Drittel-, Viertel-, Fünftel- und Sechsteltonklavier), die in Mexiko stehen und dort restauriert werden sollten, bevor man Schneiders Werk darauf spielen kann. So ist bis heute nur das erste Stück für Halb- und Dritteltonklavier aufgeführt worden.

38 Brief von Urs Peter Schneider an Roman Brotbeck vom 22.11.2016.

39 Schneider: Teich mit zwei Schwänen.

40 Brief von Urs Peter Schneider an Roman Brotbeck vom 22.11.2016.

41 Ebd. Teich mit zwei Schwänen wurde vom Trio Absolut mit Bettina Boller (Violine), Judith Gerster (Violoncello) und Stefka Perifanova (Klavier) am 7. April 2017 in Plovdiv (Bulgarien) uraufgeführt, die Schweizer Erstaufführung erfolgte durch dasselbe Ensemble am 28. Mai 2017 in Bern. Im Herbst desselben Jahres folgte unter dem Titel Der Teich Hommage an Robert Walser eine mit Text erweiterte Aufführung in Biel, in der Markus Amrein alle Rollen und die verschiedenen Szenenanweisungen des Dramoletts sprach. Hier spielten Orsolya Sepsi (Violine), Ellen Fallowfield (Violoncello) und Judith Wegmann (Klavier).

Brief von Urs Peter Schneider an Roman Brotbeck vom 08.07.2016. 


\section{0}
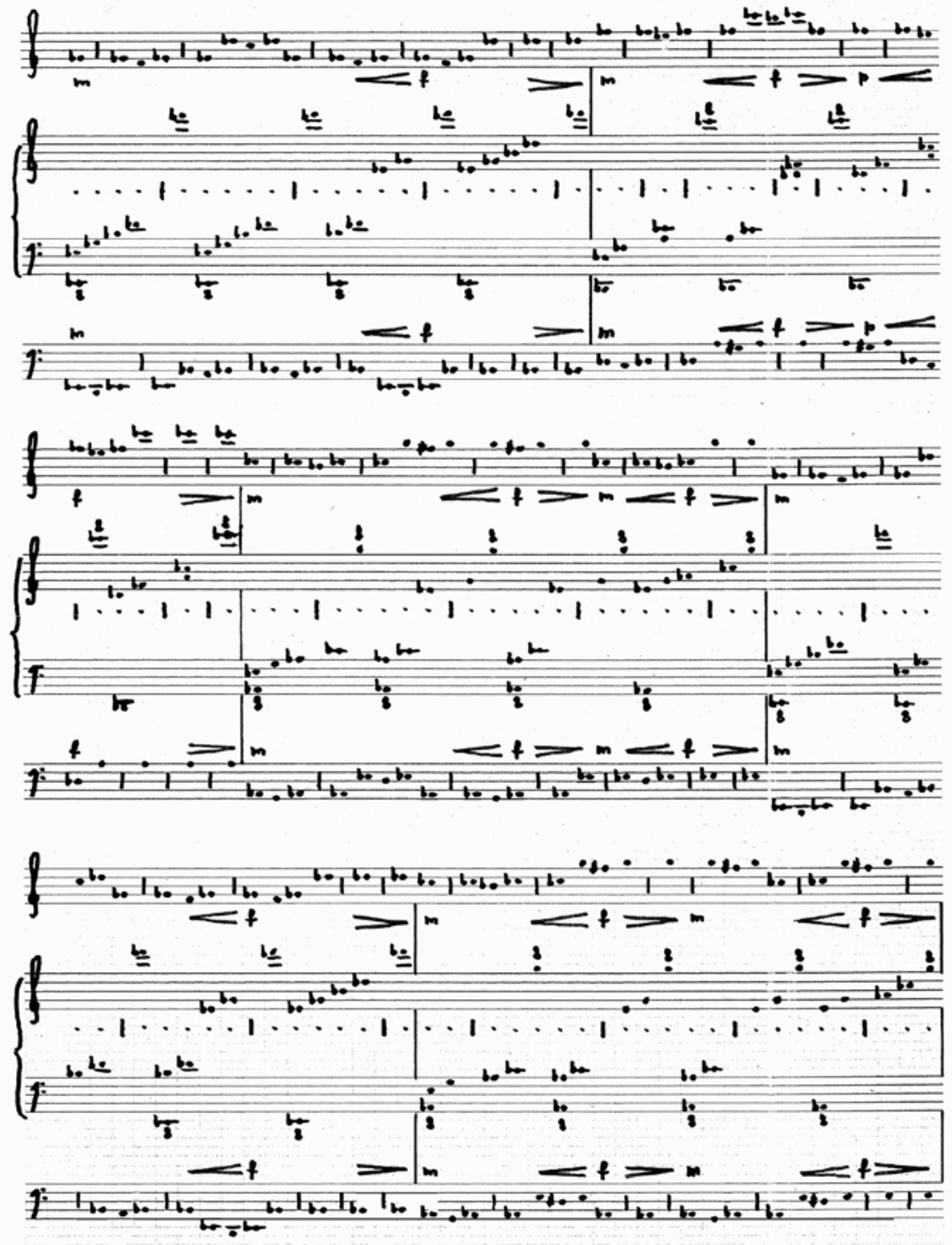

Abb. 14 Urs Peter Schneider: Teich mit zwei Schwänen (2016), Partitur, S. o 